\title{
Logical Network Mapping With Content Connectivity Against Multiple Link Failures in Optical Metro Networks
}

\author{
G. Le ${ }^{1}$, A. Marotta ${ }^{2}$, S. Ferdousi ${ }^{1}$, S. Xu ${ }^{3}$, Y. Hirota ${ }^{3}$, Y. Awaji ${ }^{3}$, Massimo Tornatore ${ }^{1,4}$, Biswanath Mukherjee ${ }^{1}$ \\ ${ }^{1}$ University of California, Davis, USA, ${ }^{2}$ University of L'Aquila, Italy, NICT, Japan ${ }^{3}$, Politecnico di Milano ${ }^{4}$, Italy \\ E-mail: dgle@ucdavis.edu
}

\begin{abstract}
Network connectivity has been the traditional metric for network survivability against failures. In case of a disaster, network connectivity may not always be guaranteed due to multiple link failures. With the shifting service paradigm towards cloud computing/storage, some network services can still be provided if a content replica is available in all disconnected network segments. As a result, content connectivity has been introduced as an additional metric for network survivability under disasters. Content connectivity is defined as the reachability of content from every node in a logical topology under a given failure scenario. In this work, we investigate the content-connectivity problem in optical metro networks in the case of multiple $(n)$ link failures. We consider the problem of mapping a logical topology over an optical metro network such that every node in the logical topology can reach at least one data center hosting the content after $n$-link failures. We formulate the problem as an integer linear program to minimize total network resource usage. We provide a cost comparison between content connectivity and network connectivity under typical failure scenarios.
\end{abstract}

Index Terms-Content connectivity, optical metro networks, data centers, survivable mapping, $n$-link failures

\section{INTRODUCTION}

With increasing demand for high-bandwidth and lowlatency services, the metro network segment is gaining more importance and functionalities. Several, especially cloud, services are now hosted directly in data centers located in the metro areas so that, in today's telecom networks, $75 \%$ of total traffic is terminated within the metro area [1]. Reliability in metro access networks is emerging as a stringent requirement and its importance is expected to increase with adoption of ultra-reliable low latency services in 5G communications.

Since the Internet was originally designed to provide end-toend communication, Network Connectivity (NC) (i.e., reachability of every network node from all other nodes) has been traditionally used as the main metric for survivability against failures. Unfortunately, in case of disasters, multiple links may be simultaneously interrupted; and providing NC in such conditions can be very costly, or even infeasible. Also, networks are becoming more content centric. Globally, $72 \%$ of all Internet traffic will cross Content Delivery Networks (CDNs) by 2022, scaling up from 56\% in 2017 [1]. Thus, several content-based services can be provided if a content replica is available in all disconnected network portions. To model this evolving reliability requirement, Content Connectivity (CC) has been introduced as an additional metric to measure network survivability [2]. CC is defined as the reachability of content from every node in a logical topology under failure scenarios. This metric is considered useful under large-scale failures as disasters, while $\mathrm{NC}$ will probably remain the default choice for smaller failure scenarios (such as single failures).

Some research on CC has already been conducted. In [2], the authors solved the $\mathrm{CC}$ problem against single-link failures. In [3], the authors extended the $\mathrm{CC}$ problem to doublelink failure scenarios. In both works, the $\mathrm{CC}$ problem was examined for backbone optical networks with mesh topologies.

In this work, we address the more general problem of CC against $n$-link failures focusing on optical metro networks where the physical topology consists of interconnected rings. We aim to provide protection to a logical topology mapped over a physical optical network. Our contributions can be summarized as follows. First, we propose a problem formulation that is more scalable than the ones in [2] and [3]. By improving scalability, our work can not only cope with larger networks with a higher number of nodes and links but can also concurrently preserve problem optimality. Second, our formulation generalizes the CC problem to an arbitrary number of link failures. The motivation of this method is to better prepare networks for large-scale failure scenarios as disasters.

The study is structured as follows. In Section II, we elaborate on the CC problem in optical metro networks. In Section III, we formulate the CC problem as an Integer Linear Program (ILP). Numerical results are shown in Section IV. Finally, we conclude in Section V.

\section{Content Connectivity Against $n$-Link FAILURES (CC- $n$ ) IN OptiCAL Metro Networks}

We consider the graph $G_{P}\left(V_{P}, E_{P}\right)$ to represent a physical topology, where $V_{P}$ and $E_{P}$ are the set of physical nodes and the set of bidirectional physical links. The physical nodes are equipped with wavelength converters, leaving the extension to the case of wavelength continuity as future work. The graph $G_{L}\left(V_{L}, E_{L}\right)$ denotes a logical topology with $V_{L}$ and $E_{L}$ being the set of logical nodes and the set of bidirectional logical links. $D$ is the set of Data Centers (DCs) in the logical topology, $D \subset V_{L}$. We assume the required content is available at all DCs. Our objective is to map the logical topology over the physical topology such that every logical node not hosting a DC can reach at least one DC for content after failures on $n$ 
distinct physical links. Logical nodes hosting DCs are contentconnected independently of physical link failures. We can now define necessary conditions for CC- $n$ existence.

Theorem 1. Given $G_{P}\left(V_{P}, E_{P}\right), G_{L}\left(V_{L}, E_{L}\right)$, and $D \subset V_{L}$, to find the mapping of $G_{L}$ over $G_{P}$ that guarantees $C C-n$, the following conditions must be satisfied:

- each logical node $s \in V_{L}-D$ has a nodal degree $\delta(s) \geq$ $n+1$, and

- each physical node $i \in V_{P}: i=s$ has a nodal degree $\delta(i) \geq n+1$.

A cut is the partition of a graph $G(V, E)$ into two disconnected parts and divides $V$ into two disjoint sets of nodes $S$ and $V-S$. Each cut defines a cutset, $C(S, V-S)$, which is the set of links with one endpoint in $S$ and the other in $V-S$. By definition of cutset, it can be derived that, after removal of all links in a cutset, the graph becomes disconnected. This latter property is also known as Menger's theorem [4], which can be applied to the CC problem against an arbitrary number of physical link failures as follows.

Let $f_{i j}^{s t}$ be a binary variable and $f_{i j}^{s t}=1$ if logical link $s t$ is mapped over physical link $i j$, and zero otherwise. We also assume traffic from $s$ to $t$ is unsplittable. The following theorem gives a necessary and sufficient condition for the mapping of a logical topology over a physical topology to be CC- $n$.

Theorem 2. Given $G_{P}\left(V_{P}, E_{P}\right), G_{L}\left(V_{L}, E_{L}\right), D \subset V_{L}$, let $\mathcal{P}_{n}=\left\{\left\{P_{n}^{k}\right\}:\left|\left\{P_{n}^{k}\right\}\right|=n,\left\{P_{n}^{k}\right\} \subset E_{P}\right\}$ be the set of all possible combinations of $n$ distinct physical links, and $C_{C C}=\left\{C_{C C}^{l}\left(S_{l}, V_{L}-S_{l}\right): S_{l} \cap D=\varnothing\right\}$ be the set of logical topology content-connected cutsets where the removal of all logical links in each cutset $C_{C C}^{l}$ disconnects $G_{L}\left(V_{L}, E_{L}\right)$ and divides $V_{L}$ into two disjoint sets with one set without DCs, the mapping of $G_{L}$ over $G_{P}$ is CC-n if and only if

$$
\sum_{\substack{i j \in P^{k} \\ s t \in C_{C C}^{l}}} f_{i j}^{s t} \leq\left|C_{C C}^{l}\right|-1, \forall P_{n}^{k} \in \mathcal{P}_{n}, \forall C_{C C}^{l} \in C_{C C} .
$$

Here, |.| is the set cardinality operation. The above condition requires that there does not exist a set of $n$ physical links whose removal leaves the logical topology disconnected with at least one segment without DC connection. In the following section, we formulate the CC- $n$ problem as an ILP.

\section{MATHEMATICAL FORMULATION}

The CC- $n$ problem can be formulated in the following ILP. Inputs and variables

- $G_{P}\left(V_{P}, E_{P}\right), G_{L}\left(V_{L}, E_{L}\right), n, D, \mathcal{P}_{n}, C_{C C}$, and $f_{i j}^{s t}$ have been introduced in Section II.

- $W$ is number of wavelengths per physical link.

- $F_{i j}$ is number of fibers on physical link $i j$.

Objective function

$$
\min \sum_{i j \in E_{P}, s t \in E_{L}} f_{i j}^{s t}
$$

\section{Subject to:}

$$
\begin{aligned}
& \sum_{s t \in E_{L}} f_{i j}^{s t} \leq F_{i j} \times W, \quad \forall i j \in E_{P} \\
& \sum_{j: j i \in E_{P}} f_{j i}^{s t}-\sum_{j: i j \in E_{P}} f_{i j}^{s t}=\left\{\begin{array}{ll}
-1 & \text { if } i=s \\
1 & \text { if } i=t \\
0 & \text { otherwise }
\end{array}\right. \text {, } \\
& \forall i \in V_{P}, \forall s t \in E_{L} \\
& \sum_{\substack{i j \in P_{n}^{k} \\
s t \in C_{C C}^{l}}} f_{i j}^{s t} \leq\left|C_{C C}^{l}\right|-1, \forall P_{n}^{k} \in \mathcal{P}_{n}, \forall C_{C C}^{l} \in C_{C C}
\end{aligned}
$$

Eqn. (1) is the objective function that minimizes network resource usage in terms of total number of wavelength channels. Eqn. (2) ensures that the mapping of the logical topology over the physical topology does not exceed each physical link capacity. Eqn. (3) enforces flow conservation for every logical link. Eqn. (4) imposes CC- $n$ on the mapping of $G_{L}\left(V_{L}, E_{L}\right)$ over $G_{P}\left(V_{P}, E_{P}\right)$. One can notice that $f_{i j}^{s t}=1$ implies that a failure on the physical link $i j$ disrupts the logical link st. Thus, Eqn. (4) ensures that failures on any combination of $n$ physical links do not disconnect all logical links in every content-connected cutset $C S_{l}$. Namely, at least one logical link in each content cutset $C S_{l}$ is survivable against every $n$-link failures on the physical topology. Hence, CC- $n$ is guaranteed.

The NC problem against $n$-link failures (NC- $n$ ) can be directly obtained from the CC- $n$ formulation with the set of $\mathrm{NC}$ cutsets being used as the input. The set of NC cutsets is the complete cutsets of the logical topology without DC consideration. In case of NC-1, this work is similar to [5]. Compared to [2], [3], and [5], our approach better prepares networks for an arbitrary number of physical link failures under disaster conditions.

\section{ILlustrative Numerical ExAmPLES}

In this section, we compare complexity of our problem formulation to complexity in [2] and [3]. We also evaluate

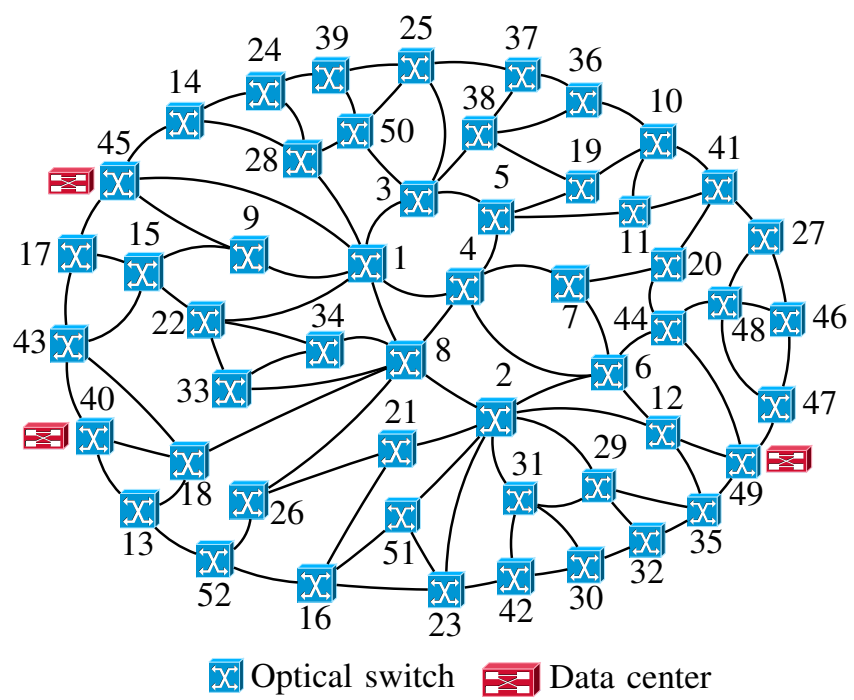

Fig. 1: Physical topology. 


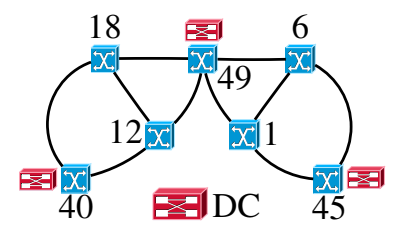

(a) Topology 1

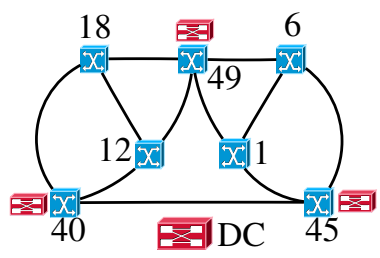

(b) Topology 2
Fig. 2: Logical topologies.

the cost in terms of number of wavelength channels of $\mathrm{CC}$ in various scenarios.

To perform our evaluation, we consider an optical metro topology as shown in Fig. 1. The physical topology consists of 52 nodes connected by rings and subrings using 98 bidirectional physical links. We consider two logical topologies as in Fig. 2a and Fig. 2b. The logical topology in Fig. 2a comprises 7 logical nodes connected by 10 bidirectional logical links. In Fig. $2 b$, the adding of a bidirectional logical link between node 40 and 45 increases the total number of bidirectional logical links to 11 . DCs are deployed at node 40,45 , and 49 . While our formulation is generic and can be applied for an arbitrary $n$, we consider $n=1$ and $n=2$ for a reasonable analysis, as the number of simultaneous link failures larger than or equal to three is rare and is left for future work.

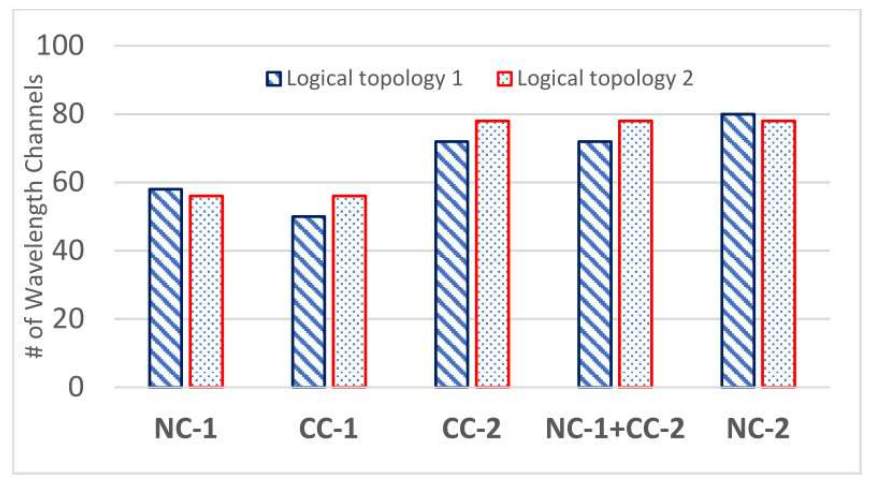

Fig. 3: Different protection schemes.

We first compare the complexity of our approach to the works in [2] and [3]. For a fair comparison, same input data are used. Numerical values of numbers of variable and constraint are shown in Table I. Compared to [2] and [3], our approach has lower complexity in terms of the total number of variables and constraints. The number of variables for $\mathrm{CC}-1$ is reduced by a factor of 23 by adopting our approach. Most importantly, the complexity of CC-2 problem is reduced by a factor of $10^{3}$ in terms of number of variables and by a factor of 60 in terms of number of constraints.

We next compare the CC- $n$ cost in terms of the total number of wavelength channels for various protection schemes. Numerical results are shown as in Fig. 3. We summarize our observations as follows.

For logical topology $1, \mathrm{NC}$ cost is higher than $\mathrm{CC}$ cost. As in
TABLE I: Complexity Comparison, Logical topology 1.

\begin{tabular}{|c|c|c|c|c|}
\hline \multirow{2}{*}{ Scenarios } & \multicolumn{2}{|c|}{ Previous works } & \multicolumn{2}{c|}{ This work } \\
\cline { 2 - 5 } & \# Var. & \# Constr. & \# Var. & \# Constr. \\
\hline NC-1 & $3,920[5]$ & $31,932[5]$ & 3,920 & 31,932 \\
\hline CC-1 & $90,220[2]$ & $90,423[2]$ & 3,920 & 13,116 \\
\hline CC-2 & $8,116,420[3]$ & $64,297,083[3]$ & 3,920 & $1,153,836$ \\
\hline NC-1+CC-2 & $8,116,420[3]$ & $64,297,083[3]$ & 3,920 & $1,178,532$ \\
\hline NC-2 & NA & NA & 3,920 & $4,822,956$ \\
\hline
\end{tabular}

Fig. 3, NC-1 utilizes 8 wavelength channels more than CC-1. This is because NC imposes stricter conditions. Indeed, failure scenarios where $\mathrm{NC}$ is not guaranteed but $\mathrm{CC}$ is still guaranteed can be found. Furthermore, CC-2 is more expensive than CC-1. CC-2 uses 72 wavelength channels while CC-1 uses only 50 wavelength channels. Noticeably, NC-1+CC-2 cost is equal to CC-2 cost. With a careful problem formulation, we can guarantee $\mathrm{NC}-1$ and $\mathrm{CC}-2$ with no additional cost compared to CC- 2 only. These results are consistent with those in [3].

However, for logical topology 2, as in Fig. 3, CC-1 and CC2 costs are equal to NC-1 and NC-2 costs, respectively. We demonstrate that there are scenarios where $\mathrm{CC}$ cost is equal to $\mathrm{NC}$ cost. In other words, $\mathrm{CC}$ cost is not always lower than $\mathrm{NC}$ cost. This observation provides a direction for future work on generalizing the scenarios in which: a) $\mathrm{CC}$ cost is lower than $\mathrm{NC}$ cost, b) $\mathrm{CC}$ cost is equal to $\mathrm{NC}$ cost, and c) $\mathrm{NC}$ is not possible but $\mathrm{CC}$ can be guaranteed.

\section{CONClusion}

In this work, we investigated the problem of ensuring $\mathrm{CC}$ against an arbitrary number of physical link failures. We first examined necessary conditions to provide $\mathrm{CC}-n$ and then proposed a solution to the CC- $n$ problem. We formulated the CC- $n$ problem as an ILP with lower complexity compared to previous works. The numerical results show that, depending on a certain scenario, CC- $n$ cost may be lower than or equal to NC- $n$ cost. A possible future extension of this work would be to generalize the $\mathrm{CC}$ problem with regard to solution feasibility and the relative cost between $\mathrm{CC}$ and $\mathrm{NC}$.

\section{ACKNOWLEDGEMENT}

This work is supported by NSF/JUNO2-1818972 project.

\section{REFERENCES}

[1] T. Muciaccia and V. M. N. Passaro, "Future scenarios for software-defined metro and access networks and software-defined photonics," Photonics, vol. 4, no. 1, Jan. 2017.

[2] M. F. Habib, M. Tornatore, and B. Mukherjee, "Fault-tolerant virtual network mapping to provide content connectivity in optical networks," Proc. IEEE/OSA OFC, Mar. 2013.

[3] A. Hmaity, F. Musumeci, and M. Tornatore, "Survivable virtual network mapping to provide content connectivity against double-link failures," Proc. 12th DRCN, Mar. 2016.

[4] R. Diestel, "Graph Theory, 5th Edition," Springer: Graduate Texts in Mathematics, 2017.

[5] E. Modiano and A. Narula-Tam, "Survivable lightpath routing: a new approach to the design of WDM-based networks," IEEE Journal on Selected Areas in Comm., vol. 20, no. 4, pp. 800-809, May 2002. 